\title{
Arterial Stiffness in Chronic Kidney Disease and End-Stage Renal Disease
}

\author{
Gerard M. London \\ Department of Nephrology, Hospital Manhes, Fleury-Mérogis, France
}

\section{Keywords}

Aging · Arterial stiffness · Microcirculation · Inflammation

\begin{abstract}
Accelerated ageing is observed in patients with chronic kidney disease (CKD)/end-stage renal disease. Premature vascular aging and arterial stiffening are the most characteristic features of this "progeria" that is already observed in those with the early stages of CKD. Aortic stiffening is associated with high characteristic impedance, left ventricular hypertrophy, decreased coronary perfusion, and is a strong prognostic marker of mortality and cardiovascular morbidity. With aging, the arterial stiffening is more pronounced in the aorta and central arteries than in peripheral conduit arteries. This leads to progressive decrease and inversion of the arterial stiffness gradient and systemic reflection coefficient, leading to less protection of the microcirculation in the event of high-pressure transmission towards it Arterial stiffening is multifactorial with systemic microinflammation being one of the most important associated factors primarily associated with vascular calcifications.
\end{abstract}

(c) 2018 S. Karger AG, Basel

E-Mail karger@karger.com www.karger.com/bpu

\section{Introduction to Pathophysiology and Methodology}

Epidemiological and clinical cross-sectional studies emphasized the roles of arterial remodeling and arterial stiffness as independent cardiovascular risk factors and predictors of all-cause and cardiovascular death in several diseases [1-3]. Arterial stiffness describes the arterial pressure response to stroke volume changes. During ventricular contraction, a part of the stroke volume is stored in the aorta and central arteries, distending and stretching the arterial walls (raising the blood pressure [BP]). Distension and stretching divert part of the cardiac work. To limit this part of cardiac work, the energy necessary to distend the artery should be low (for a given stroke volume, the pressure increase should be low). The efficiency of this function depends on artery stiffness. When stiffness is mild, the arterial wall opposes low resistance to distension and the pressure effect is minimized. In addition, arterial stiffness determines the propagation velocity of the pressure wave in the arterial segments and tree, that is, pulse wave velocity (PWV). PWV assesses the stiffness of an artery as a hollow structure and according to Moens and Korteweg equation, it depends on the ar- 
Fig. 1. Correlations between age and carotid-to-femoral pulse wave velocity (PWV) in control populations (in red) and ESRD patients (green). The slopes ( $\beta$ coefficients) are significantly increased in ESRD $(p<$ 0.00001). London GM Manhes Hospital registry.

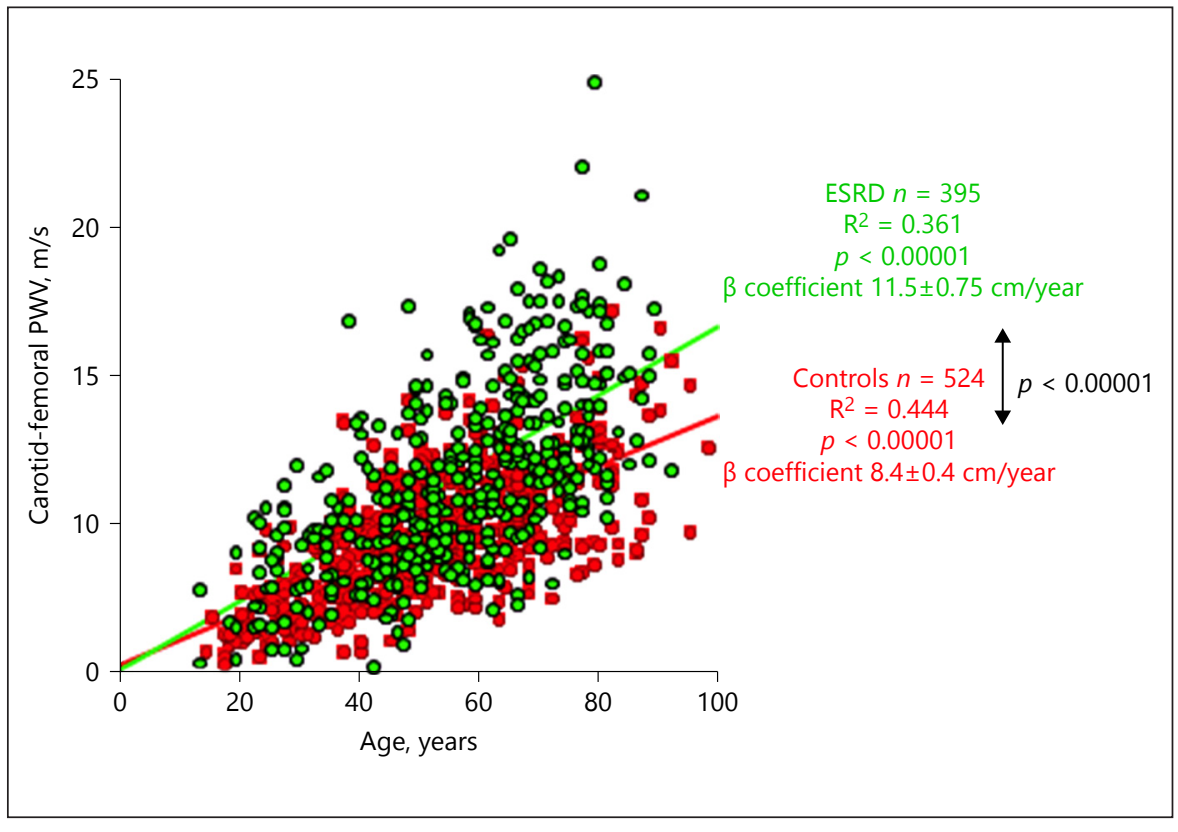

tery geometry (wall thickness, h; radius, $\mathrm{r}$ ), arterial biomaterials elastic properties (E-incremental elastic modulus), and tissue density ( $\delta): \mathrm{PWV}^{2}=\mathrm{E} \cdot \mathrm{h} / 2 \mathrm{r} . \delta$ [4]. Due to arterial system heterogeneity, PWV increases progressively from the ascending aorta to the peripheral muscular conduit arteries, generating a progressive stiffness gradient $[5,6]$. Although PWV can be measured on any artery or between any arterial sites, only carotid-to-femoral PWV ("aortic") has been shown to have a predictive value for morbidity and mortality $[5,6]$. The measurement of carotid-to-femoral PWV is considered the gold standard as a large body of evidences demonstrated the association of aortic stiffness with cardiovascular disease and mortality in various populations, independently of traditional risk factors $[1-3,6,7]$.

Due to arterial system heterogeneity, PWV increases progressively from the ascending aorta to the peripheral muscular conduit arteries, generating a stiffness gradient that regulates pressure transmission along the arterial tree and to the microcirculation $[4,5,8-10]$. The stiffness gradient, together with aortic geometry changes (tapering), local arterial branching, and lumen narrowing, creates impedance mismatches, causing partial reflections (reflected waves) of the pressure wave generated in the aorta (forward/incident) [4]. Forward and reflected pressure waves overlap. The final amplitude and shape of the pulse pressure wave along the arterial tree are determined by the timing between these component waves. Arterial stiffening disrupts the desirable timing principally in the aorta and central arteries. With increased PWV, the reflected waves return earlier during systole, amplifying aortic and ventricular pressures during systole, and reducing aortic pressure during diastole [4, 5, 8-10]. The principal consequences are increased myocardial pressure load (left ventricular hypertrophy), higher oxygen consumption, and decreased diastolic BP and coronary blood flow $[5,11,12]$.

\section{Arterial Stiffness in End-Stage Renal Disease (Chronic Kidney Disease 5D)}

Cardiovascular disease is a major cause of morbidity and mortality in patients with chronic kidney disease (CKD) or end-stage renal disease (ESRD) [13-15]. Premature aging and early vascular aging is the most characteristic arterial change observed in ESRD $[14,15]$ (Fig. 1). Premature vascular aging and arterial stiffening are observed with the progression of CKD [16-18]. The most characteristic arterial change observed is the arterial stiffening, more pronounced in the aorta than in peripheral arteries $[6,19]$. This condition is responsible for the reduction of the arterial stiffness gradient and impedance mismatch with diminished capacity to lower pulsatile pressure transmission to the peripheral microcirculation $[8,15]$. In ESRD, the aortic PWV was shown to have an independent predictive power for all-cause and CV mortality $[1-3,20,21]$. Increasing arterial stiffness is repre- 
Fig. 2. Blood pressure-adjusted correlations between age and aortic pulse wave velocity (PWV) in ESRD patients according to serum high-sensitive CRP. Aortic aging is significantly increased in the presence of microinflammation $(p<0.00001)$. London GM Manhes Hospital registry.

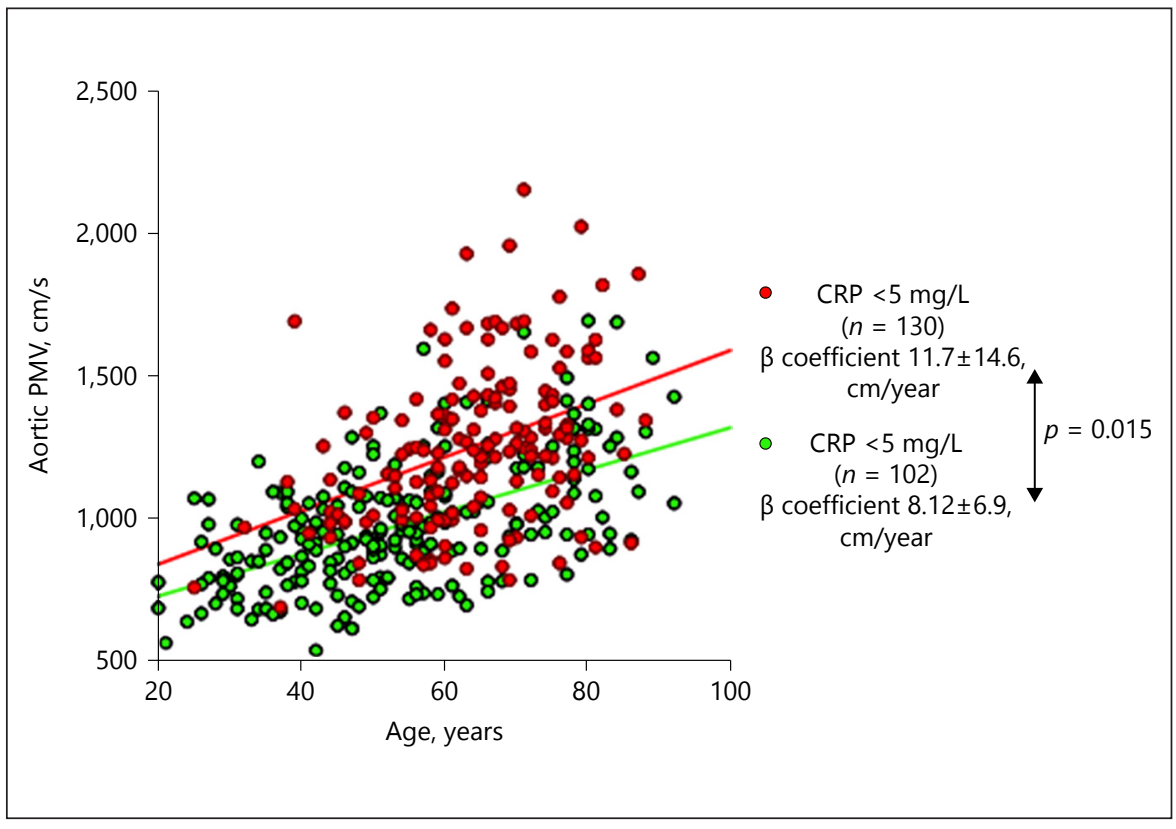

sentative of the arterial aging process. Nevertheless, a significant interaction exists between aortic PWV and age, and compared to the general population, in ESRD, the difference in the mortality rate gradually decreases with aging [22]. In a recent study [20] aimed to analyze the interplay between age and PWV in ESRD, it has been shown that the predictive value of aortic PWV on cardiovascular and all-causes mortality was significant in the younger population. An aortic PWV $>12 \mathrm{~m} / \mathrm{s}$ provides important prognostic information in ESRD patients $<60$ years of age, whereas in older patients, its prognostic relevance is lost. Patients with ESRD have a 10 to 30 -fold higher cardiovascular mortality rate compared to the general population. This difference is highly accentuated in younger individuals in whom mortality rates can reach up to a value that is 500 -fold higher [22].

Arterial remodeling and premature vascular aging are already observed in the early stages of CKD and its progression [16-19]. Compared with normotensive and hypertensive controls, patients with CKD stages $2-5$ had significantly higher aortic PWV. The arterial stiffening in earlier stages of CKD and increased risk in ESRD populations $<60$ years suggest that PWV measurement should be performed in younger CKD and ESRD patients in order to gain prognostic information and to potentially guide early interventions before the occurrence of irreversible structural changes. In CKD and ESRD, stiffening is associated with increased arterial diameters and intima-media thickness, and alterations of the intrinsic elas- tic properties of arterial walls (E), namely, calcification of elastic lamellae, elastinolysis, increased collagen content, collagen cross-linking, apoptosis and rarefaction of vascular smooth muscle cells, and inflammation [5, 23-29]. In ESRD patients, arterial stiffness was associated with arterial calcifications and worsens with increasing calcifications. Chronic microinflammation is a characteristic condition in ESRD and plays a role in arterial aging [14, 15]. Figure 2 shows that age-associated aortic stiffening is more pronounced in subjects with increased highly sensitive CRP. Inflammatory cytokines are potent activators of «osteogenic» phenotype of smooth muscle cells and arterial calcifications [26]. Several noncardiovascular diseases, such as rheumatoid arthritis or systemic lupus erythematosus, are associated with increased aortic stiffening, underscoring the role of inflammation in the rigidity of large arteries [27-29]. The association of arterial stiffening and inflammation was also demonstrated in essential hypertension through the relationships between arterial stiffness and either tumor necrosis factor- $\alpha$, interleukin-6, or highly sensitive CRP [29].

Besides age, arterial stiffness is also influenced by arterial BP. The association of $\mathrm{BP}$ and stiffness are ambiguous, since stiffening can directly influence the BP profile with higher systolic and pulse pressures, but in parallel, the increased BP-related distension of arterial walls can increase stiffness [4]. In essential hypertensive subjects, the arterial stiffness is in part due to higher distending stress applied on arterial walls. BP reduction should normally decrease 
PWV, which in this case is "pressure dependent." In hemodialysis subjects, Guérin et al. [30] examined the patients' outcomes according to aortic PWV response to antihypertensive therapies (nonpharmacological interventions and antihypertensive drugs) and reduction in BP. Over a mean follow-up of 50 months, surviving patients were characterized by simultaneous decrease in aortic PWV and BP, that is, "pressure-dependent stiffness." In non-survivors, despite a similar reduction in BP, the aortic PWV steadily increased, that is, "pressure-independent stiffening." This observation suggested the hypothesis that modification of arterial stiffness and not BP could be the risk factor associated with the prognosis and outcomes. The recent study by Sarafidis et al. [19] supports this hypothesis. In hemodialysis patients, authors compared the value of 48-h ambulatory BP (brachial and central) and 48-h aortic PWV measures for major cardiovascular outcomes and all-cause mortality. In a multivariate analysis, only 48-h PWV measure was the vascular parameter independently associated with the increased risk of cardiovascular events and mortality, supporting that arte- rial stiffness is the prominent cardiovascular risk factor in hemodialysis. When the arterial stiffness is pressure dependent, BP reduction should normally contain rigidification. Nevertheless, evidences are still required for therapies that should reflect a real diminution of arterial wall rigidity, independent of BP effects.

Many studies emphasized the role of arterial stiffness in the development of cardiovascular diseases, Arterial rigidity is closely associated with vascular aging, and premature vascular aging is observed with CKD progression and in ESRD. Arterial hardening is of a multifactorial origin, with extensive arterial calcifications representing a major covariate. With aging, the stiffening is more pronounced in the aorta than peripheral conduit arteries, leading to the disappearance or inversion of the arterial stiffness gradient with diminished protection of the microcirculation against high pulsatile-pressure transmission. Various nonpharmacological or pharmacological interventions can modestly slow arterial stiffness but presently treatment methods that are able to prevent stiffness mainly include antihypertensive drugs.

\section{References}

1 Blacher J, Guérin AP, Pannier B, et al: Impact of aortic stiffness on survival in end-stage renal disease. Circulation 1999;99:2434-2439.

2 Laurent S, Boutouyrie P, Asmar R, et al: Aortic stiffness is an independent predictor of allcause and cardiovascular mortality in hypertensive patients. Hypertension 2001;37:12361241.

3 Cruickshank K, Riste L, Anderson SG, et al: Aortic pulse-wave velocity andits relationship to mortality in diabetes and glucose intolerance: an index of vascular function. Circulation 2002;106:2085-2090.

4 O'Rourke MF: Principles and definitions of arterial stiffness, wave reflections and pulse pressure amplification; in Safar ME, O’Rourke MF(eds): Handbook of Hypertension (series edts Birkenha"ger WH, Reid JL). Arterial Stiffness in Hypertension. Amsterdam, Elsevier, 2006, vol. 23, pp 3-20.

5 Briet M, Boutouyrie P, Laurent S, London GM: Arterial stiffness and pulse pressure in CKD and ESRD. Kidney Int 2012;82:388400.

6 Pannier B, Guérin AP, Marchais SJ, et al: Stiffness of capacitive and conduit arteries: prognostic significance for end-stage renal disease patients. Hypertension 2005;45:592-596.

7 Boutouyrie P, Tropeano AI, Asmar R, et al: Aortic stiffness is an independent predictor of primary coronary events in hypertensive patients: a longitudinal study. Hypertension 2002;39:10-15.
8 Mitchell GF: Effects of central arterial aging on the structure and function of the peripheral vasculature: implications for end-organ damage. J Appl Physiol 2008;105:1652-1660.

9 London GM, Pannier B: Arterial functions: how to interpret the complex physiology. Nephrol Dial Transplant 2010;25:3815-3823.

10 O'Rourke MF, Safar MR: Relationship between aortic stiffening and microvascular disease in brain and kidney: cause and logic of therapy. Hypertension 2005;46:200-204.

11 Avolio AP, Chen SG, Wang RP, et al: Effects of aging on changing arterial compliance and left ventricular load in a northern Chinese urban community. Circulation 1983;68:50-58.

12 Zoungas S, Cameron JD, Kerr PG, et al: Association of intima-media carotid thickness and indices of arterial stiffness with cardiovascular disease outcome in CKD. Am J Kidney Dis 2007;50:622-630.

13 Sarnak MJ, Foley RN: Cardiovascular mortality in the general population versus dialysis: a glass half full or empty? Am J Kidney Dis 2011;58:4-6.

14 Kooman JP, Kotanko P, Schols AM, et al: Chronic kidney disease and premature ageing. Nat Rev Nephrol 2014;10:732-742.

15 London GM, Safar ME, Pannier B: Aortic aging in ESRD: structural, hemodynamic, andmortality mplications. J Am Soc Nephrol 2016;27:1837-1846.

16 Briet M, Bozec E, Laurent S, et al: Arterial stiffness and enlargement inmild to moderate chronic kidney disease. Kidney Int 2006;96: 350-357.

17 Kimoto E, Shoji T, Shinohara K, et al: Preferential stiffening of central over peripheral arteries in type 2 diabetes. Diabetes 2003;52: 448-452.

18 Ferreira JP, Girerd N, Pannier B, et al: High pulse-wave velocity defines a very high cardiovascular risk cohort of dialysis patients under age 60. Am J Nephrol 2017;45:72-81.

19 Sarafidis PA, Loutradis Ch, Karpetas A, et al: Ambulatory pulse wave velocity is a stronger predictor of cardiovascular events and allcause mortality than office and ambulatory blood pressure in hemodialysis patients. Hypertension 2017;70:148-157.

20 Foley RN, Parfrey PS, Sarnak MJ: Clinical epidemiology of cardiovascular disease in chronic renal disease. Am J Kidney Dis 1998; 32(5 suppl 3):S112-S119.

21 Wang MC, Tsai WC, Chen JY, et al: Stepwise increase in arterial stiffness corresponding with the stages of chronic kidney disease. Am J Kidney Dis 2005;45:494-501.

22 Ford ML, Tomlinson LA, Chapman TP, et al: Aortic stiffness is independently associated with rate of renal function decline in chronic kidney disease stages 3 and 4 . Hypertension 2010;55:1110-1115.

23 Amann K, Neususs R, Ritz E, et al: Changes of vascular architecture independent of blood pressure in experimental uremia. Am J Hypertens 1995;8:409-417. 
24 London GM, Guérin AP, Marchais SJ, et al: Arterial media calcification in end-stage renal disease: impact on all-cause and cardiovascular mortality. Nephrol Dial Transplant 2003; 18:1731-1740.

25 Raggi P, Bellasi A, Ferramosca E, et al: Association of pulse wave velocity with vascular and valvular calcification in hemodialysis patients. KidneyInt 2007;71:802-807.
26 Shao JS, Cheng SL, Towler DA: Inflammation and the osteogenic regulation of vascular calcification: a review and perspective. Hypertension 2010;55:579-592.

27 Klocke R, Cockcroft JR, Taylor GJ, et al: Arterial stiffness and central bloodpressure, as determined by pulse wave analysis, in rheumatoid arthritis. Ann Rheum Dis 2003;62:414-418.

28 Mahmud A, Feely J: Arterial stiffness is related to systemic inflammation in essential hypertension. Hypertension 2005;46:1118-1122.
29 Maki-Petaja K, Hall F, Booth A, et al: Rheumatoid arthritis is associated with increased aortic pulse-wave velocity, which is reduced by antitumor necrosis factor-alpha therapy. Circulation 2006;114:1185-1192.

30 Guérin A, Blacher J, Pannier B, et al: Impact of aortic stiffness attenuation on survival of patients in end-stage renal failure. Circulation 2001;103:987-992. 